\title{
Structure-Behavior Coalescence Design to Improve the Modifiability of Home Care IoT System
}

\author{
Shuh-Ping Sun ${ }^{1}$, William S. Chao ${ }^{2}$ \\ ${ }^{1}$ Department of Digital Media Design, I-Shou University, TAIWAN \\ ${ }^{2}$ SBC Architecture International@, U.S.A \\ Email: ${ }^{1}$ spsun@isu.edu.tw, ${ }^{2}$ architectchao@gmail.com
}

Received: May 30, 2021. Received: November 8, 2021. Accepted: November 27, 2021. Published: December 9, 2021.

\begin{abstract}
Modifiability improvement is a key factor in the successful Home Care IoT System (HCIS) systems development. It includes disciplined system layering (DSL), well-defined components (WDC), published interface (PI), and well-defined behavior (WDB) which represent the four main factors that enhance the modifiability of HCIS. Structure-Behavior Coalescence (SBC) method uses three fundamental diagrams: a) framework diagram, b) component operation diagram, and c) interaction flow diagram to accomplish the design of HCIS. Through framework diagram, Structure-Behavior Coalescence design of HCIS demonstrates tremendous effects of disciplined system layering. Through component operation diagram, Structure-Behavior Coalescence design of HCIS demonstrates large effects of well-defined components and published interfaces. Through interaction flow diagram, Structure-Behavior Coalescence design of HCIS demonstrates tremendous effects of well-defined behaviors. Structural Equation Modeling (SEM) refers to a diverse set of unrelated computer algorithms and statistical methods, which are suitable for constructing networks for analysis. Applied SEM method can verify that Structure-Behavior Coalescence design is be able to enhance the Modifiability of HCIS.
\end{abstract}

Keywords-Home Care IoT System, Modifiability, Structure-Behavior Coalescence Design, Structural Equation Modeling.

\section{INTRODUCTION}

Home Care IoT system (HCIS) is a system designed to satisfy the remote care as well as home care service providers. HCIS covers a great deal of home care service. A Home Care IoT system consists of a group of remote care slots.

When talking about "design for Modifiability", we are talking about the design decisions in order to enable us to easily and effectively modify our system $[1,6]$. There are four main factors to enhance the Modifiability of HCIS.

(A) Disciplined System Layering (DSL). In almost all cases, a single component does not work alone. In HCIS, each component interacts with other components and depends on them to function properly. When writing tests, our ability to isolate the given component from all others dependencies is crucial. And we must think of putting mechanisms in place to enable us to do so easily.

(B) Well-Defined Components (WDC). When writing automatic unit modifications, the main difficulty faced is the need to isolate the modified components in HCIS from the rest. In order to modify a functionality of components, firstly one needs to detach it from the rest of HCIS in which it is designed to work. Once the component exists, one needs to activate the modified functionality and finish by ensuring that the resulting behavior meets our expectations. However, unless HCIS is designed specifically to enable this, in most cases, it will not be simple.

(C) Published Interfaces (PI). When writing unit modifications, we faced some problems. For example: Creating a component. In most cases a component is not meant to be created in a standalone manner, as we do when writing modified. Normally, components are created as part of an entire system. Since they depend on other components of HCIS, they make sure those components are there and working correctly. Setting these components in the modifying environment is expensive and complex. Therefore, a mechanism needs to create the modified component without creating the rest of the dependencies as well.

(D) Well-Defined Behaviors (WDB). In order to write meaningful modifications, the expected behavior must be checked. In some cases, this behavior can only be observed by viewing the result state of the component at the end of the modification. However, in many cases, the modified component has no meaningful state of its own, and its purpose is to correctly interact with other parts. In order to verify this interaction we need a way to allow it during modifying, making sure all expected interactions were carried out as they should. In order 
to write effective unit modify for HCIS, we need to effectively isolate each component, and surround it with fakes (created as part of the modified), which will enable verification of all interactions carried out by the component under modification. The ease of writing unit tests is in direct correlation to this ability.

Structure-Behavior Coalescence (SBC) method can be used to formally design the integration of systems structure and systems behavior. Structure-Behavior Coalescence design contains three fundamental diagrams: a) framework diagram, b) component operation diagram, and c) interaction flow diagram. Structure-Behavior Coalescence approach uses three fundamental diagrams: a) framework diagram, b) component operation diagram, and c) interaction flow diagram to accomplish the design of HCIS. Through framework diagram, Structure-Behavior Coalescence design of HCIS demonstrates tremendous effects of disciplined system layering. Through component operation diagram, Structure-Behavior Coalescence design of HCIS demonstrates large effects of well-defined components and published interfaces. Through interaction flow diagram, Structure-Behavior Coalescence design of HCIS demonstrates tremendous effects of well-defined behaviors.

Structural equation modeling (SEM) refers to a diverse set of unrelated computer algorithms and statistical methods that fit networks of constructs to data. SEM includes confirmatory factor analysis, path analysis, partial least squares path analysis, latent growth modeling. Structural equation models are often used to assess unobservable 'latent' constructs. They often invoke a measurement model that defines latent variables using one or more observed variables, and a structural model that imputes relationships between latent variables. In this paper, we shall use SEM to verify that the Structure-Behavior Coalescence design is indeed able to enhance the modifiability of HCIS.

This paper is organized as follows. Section 1 is the introduction. Related works are surveyed in Section 2. Section 3 explores in detail the Structure-Behavior Coalescence design of HCIS. Verifying that the Structure-Behavior Coalescence design is indeed able to enhance the modifiability of HCIS is discussed in Section 4. Section 5 is a conclusion.

\section{RELATED WORKS}

Systems design is part of the overall systems development process as reflected in the systems development life cycle. The phases of the systems development life cycle are as follows: a) project planning, b) requirements and specifications, c) design and implementation, d) verification and validation, and e) product evolution. The system life cycle applies recursively to life cycles that produce hardware and software portions of the system.

Systems design is, in the systems development process, the design and implementation phase. That is, systems design means to get a solution to furnish customers' requirements on the system. When working on the systems design, we mainly consider how to manufacture the system, but not to specify what this system is.

In general, a system is extremely complex that it consists of multiple views such as structure view, behavior view, function view, data view as shown in [9], [13], [15]. Among the above multiple views, the structure and behavior views are perceived as the two prominent ones. The structure view focuses on the systems structure which is described by components and their composition while the behavior view concentrates on the systems behavior which involves interactions among the external environment's actors and components [25]-[26].

Structure-Behavior Coalescence (SBC) method [2]-[5] provides an elegant design to integrate the systems structure and behavior within the system. A system designed by the Structure-Behavior Coalescence approach has the following characteristics: 1) it emphasizes the system's structure-behavior coalescence; 2) it is a truly integrated whole; 3 ) it is embodied in its assembled components; 4) components are interacting (or handshaking) [8], [10]-[11] with each other and the environment; and 5) it uses structural decomposition [7] rather than functional decomposition [14].

Structure-Behavior Coalescence design uses the SBC approach to formally design the integration of systems structure and systems behavior of a system. Structure-Behavior Coalescence design contains three fundamental diagrams: a) framework diagram, b) component operation diagram, and c) interaction flow diagram.

\section{STRUCTURE-BEHAVIOR COALESCENCE DESIGN OF HCIS}

Structure-Behavior Coalescence model provides an elegant way to integrate the systems structure and systems behavior of a system. Structure-Behavior Coalescence model uses the SBC process algebra to accomplish the design of HCIS.

\section{A. Structure-Behavior Coalescence Model}

\section{1) Operation-Based Interactions}

An interaction represents an indivisible and instantaneous handshake or communication between two agents. In the operation-based interaction approach as shown in Fig. 1, the caller agent (either external environment's actor or component) interacts with the callee agent (component) through the operation call or operation return interaction. In the figure, "Calculate_Age (In SSN)" is an operation_call_formula and "Calculate_Age (Out Age)" is an operation_return_formula. 


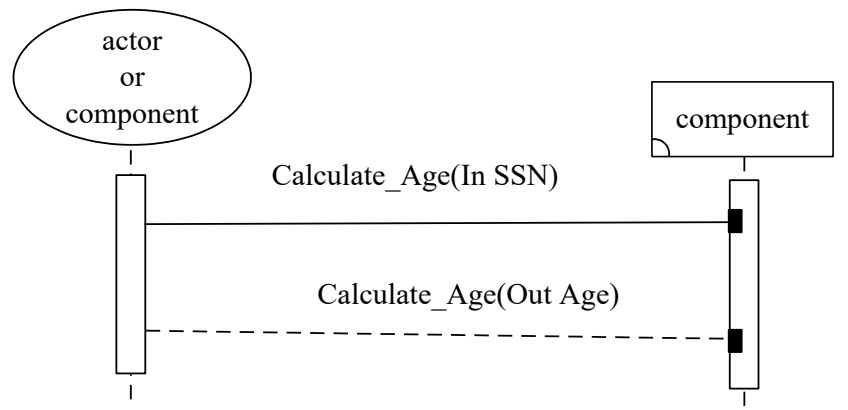

Fig.1 operation-based interactions

The external environment uses a "type-1 interaction" to interact with a component. We formally describe an operation-based value-passing type_1 interaction as a 4-tuple TYPE_1_INTERACTION $=<$ operation_call_or_return, actor, operation_call_or_return_formula, callee_component>, where "operation_call_or_return" stands for an OPERATION_CALL or OPERATION_RETURN tag, "actor" represents the name of an external environment's actor, "operation_call_or_return_formula" stands for an operation call or operation return formula and "callee_component" stands for the name of a call component as shown in Fig. 2.

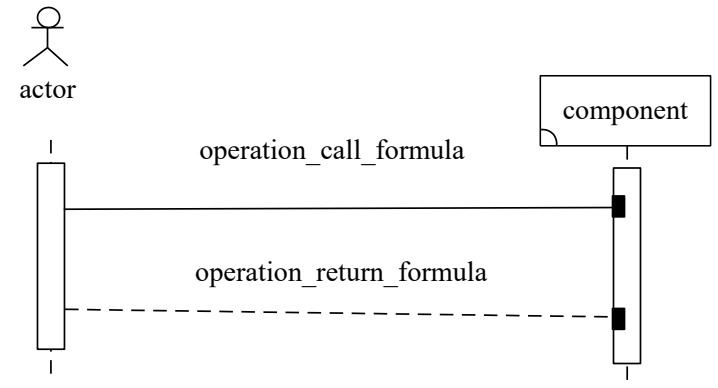

Fig.2 formal description of a type_1 interaction

Two components use a "type-2 interaction" to interact with each other. We formally describe an operation-based value-passing type 2 interaction as a 4-tuple TYPE_2_INTERACTION $=$ <operation_call_or_return, caller_component, operation_call_or_return_formula, callee_component $>$, where "operation_call_or_return" represents a OPERATION_CALL or OPERATION_RETURN tag, "caller_component" represents the name of a caller component, "operation_call_or_return_formula" represents a operation call or operation return formula and "callee_component" represents the name of a callee component as shown in Fig. 3.

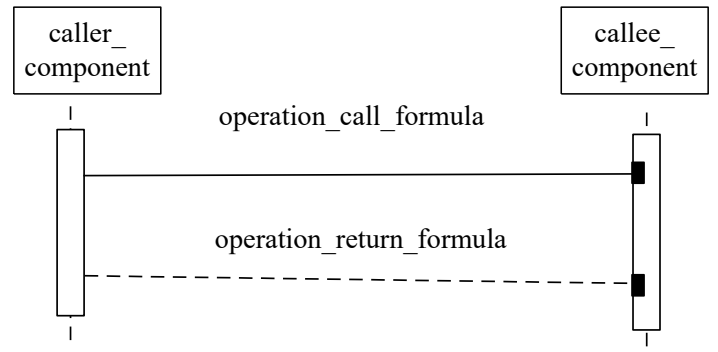

Fig. 3 formal description of a type_ 2 interaction

\section{2) Collection of All Interaction Flow Diagrams Defining a} System

In the structure-behavior coalescence model, the collection of all interaction flow diagrams (IFD) defines a system as shown in Fig. 4.

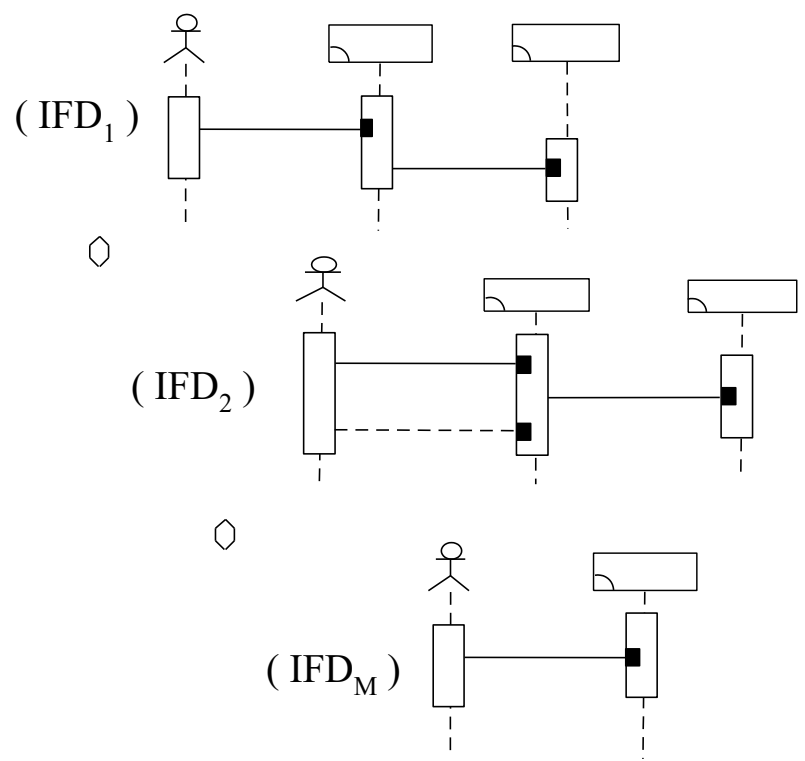

Fig. 4 collection of all interaction flow diagrams defines a system

Since the collection of all interaction flow diagrams defines a system, we formally describe a system as a collection, where " $\mathrm{x}$ " stands for the xth interaction flow diagram of this system.

\section{3) A Sequence of Interactions Defining an Interaction}

Flow Diagram

In the structure-behavior coalescence model, a sequence of interactions defines an interaction flow diagram (IFD) as shown in Fig. 5.

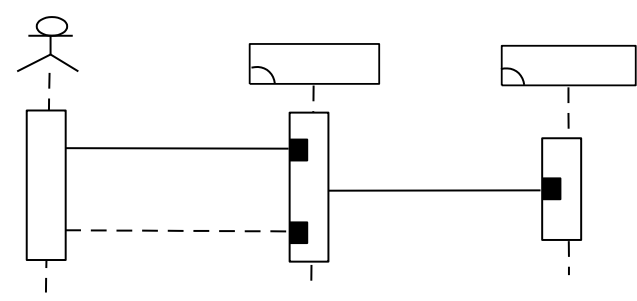

Fig. 5 a sequence of interactions defines an IFD 
Since a sequence of interactions defines an interaction flow diagram, we formally describe the xth interaction flow diagram, i.e., IFDx of the system as a sequence, where " $z$ " stands for the $\mathrm{zth}(\mathrm{z}=1$ to $\mathrm{N})$ interaction of this $\mathrm{xth}$ interaction flow diagram. Note that the first interaction, i.e., the interactionx 1 , must be type-1, while the other interactions, i.e., the interactionxz( $z=2$ to N), can be type-1 or type- 2 .

\section{4) Summary of the SBC Model}

In the SBC model, a system is formally described as a collection, and the xth interaction flow diagram, i.e., IFDx of the system is formally described as a sequence. To combine them together, we summarize that in the SBC model a system is then formally described as .

Examining the SBC model, we found that the SBC model has two parts. The first part defines the behavior of a system. The second part defines the interactions that occur in the behavior of a system.

\section{B. Structure-Behavior Coalescence Design of HCIS}

Home care, also referred to as domiciliary care, is supportive care provided in the home [12]. Care may be provided by licensed healthcare professionals who provide medical care needs or by professional caregivers who provide daily care to help to ensure the activities of daily living are met [23]-[24].

Advancements in the home care IoT system (HCIS) present enormous potential for the intensified healthcare support of senior residents at home. By using HCIS at home, senior residents are able to live independently for a longer period of time, helping to reduce costs and the need for additional caregiver resources in the process.

The SBC model uses five IFDs to accomplish the design of HCIS. Fig. 6 shows the 1st IFD which describes how each family registers with HCIS. First, actor Homecare_Provider interacts with the Home_Account_Registering_UI component through the Input_Home_Data operation call interaction, carrying the Home_Data_Form input parameter. Next, component Home_Account_Registering_UI interacts with the SBCDHCIS_Database component through the SQL_Insert_Home_Data operation call interaction, carrying the Home_Data_Query input parameter. Each family needs to register with SBCDHCIS for home care applications.

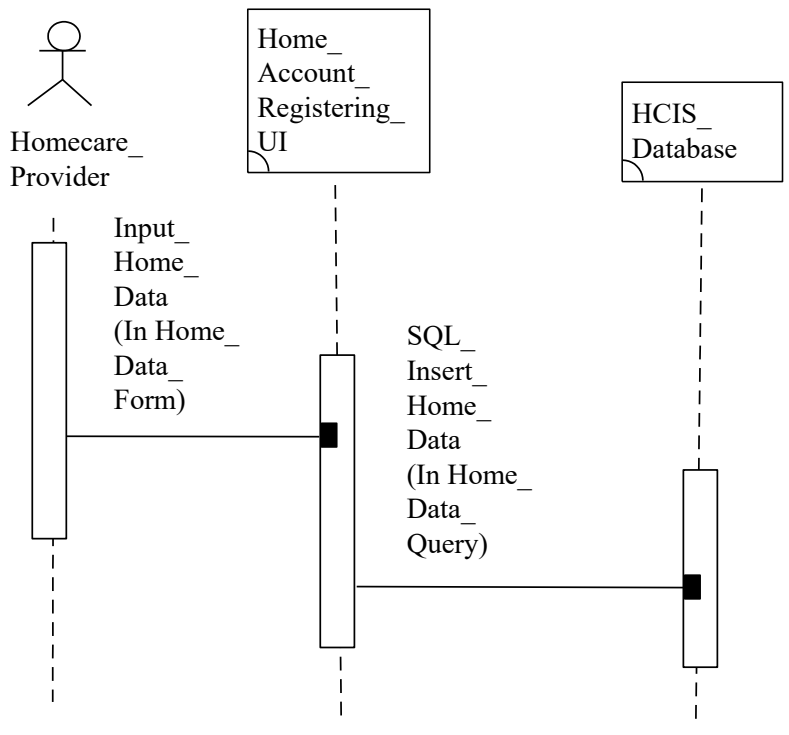

Fig. 6 each family registers with HCIS

\section{VERIFYING THE MODIFIABILITY IMPROVEMENT OF HCIS}

In this section, we use SEM to verify that Structure-Behavior Coalescence Design is really be able improve the modifiability of HCIS.

\section{A. Empirical Hypotheses}

From review literatures, and a preliminary in-depth interview with 21 systems engineering administrator, 2 groups of constructs emerge as influencers of Structure-Behavior Coalescence Design of Home Care IoT System (SBCDHCIS) and Modifiability of Home Care IoT System (MHCIS) and their relationship is shown in Fig. 7.

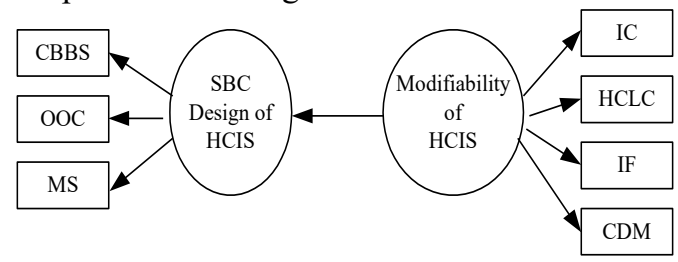

Fig. 7 Relationship of SBCDHCIS and MHCIS

Figure 4-1. Relationship Between SBCDSPIS and MSPIS

Based on the previous research mentioned above, we test the following hypotheses:

CBBS (Components as the Building Block of a System): revised as Framework Diagram (FD) positively effects on Home

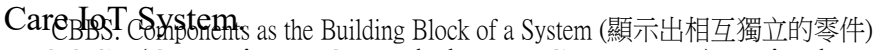

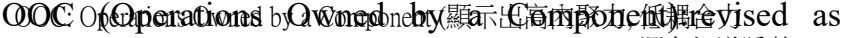

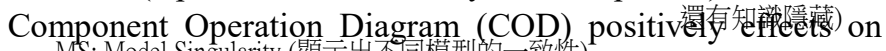
Homs: Carel Pingullarity (

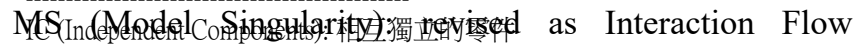

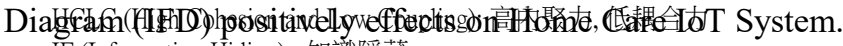

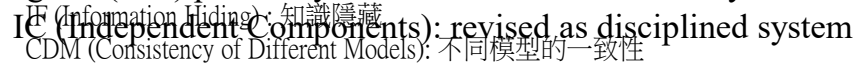


layering (DSL)

HCLC (High Cohesion and Low Coupling): revised as published interfaces $(\mathrm{PI})$

IF (Information Hiding): revised as well-defined behaviors (WDB)

CDM (Consistency of Different Models): revised as well defined components (WDC)

\section{B. Empirical Methods}

\section{1) Measures}

In the empirical method [16]-[17], 7 items capture AODSTCIS (revised as SBCDHCIS) and TSTCIS (revised as MHCIS). We measured SBCDHCIS using three-item from Kishen et al [20]. The four-item scale in Kishen et al [20] study offers a good tool to measure MHCIS. We use AMOS to implement Structural Equation Modeling shown in figure 7. Firstly Table I displays figure 7's item details together with their standard deviation and mean. According previous studies report strong psychometric properties, the experimental data from our survey as shown in Table I that's also acceptable validity and reliability scales in this empirical study.

Table I. Scale items with mean, standard deviation, and standardized loadings

\begin{tabular}{|c|l|c|c|c|}
\hline \multicolumn{2}{|c|}{ Construct } & Mean & S.D. & $\begin{array}{l}\text { Standardized } \\
\text { loadings }\end{array}$ \\
\hline FD & $\begin{array}{l}\text { Our office always uses technology } \\
\text { provided by framework diagram }\end{array}$ & 5.92 & 1.04 & 0.78 \\
\hline COD & $\begin{array}{l}\text { Our office fully utilize the functionality } \\
\text { provided by component operation } \\
\text { diagram technologies }\end{array}$ & 6.17 & 1.05 & 0.80 \\
\hline IFD & $\begin{array}{l}\text { Interaction flow diagram technologies are } \\
\text { completely ingrained in our business } \\
\text { practices }\end{array}$ & 6.00 & 0.89 & 0.73 \\
\hline TSTCIS & $\begin{array}{l}\text { Our office enjoy the result of disciplined } \\
\text { system layering }\end{array}$ & 6.03 & 0.93 & 0.88 \\
\hline DSL & $\begin{array}{l}\text { My office perceives published interfaces } \\
\text { to be a useful source of knowledge } \\
\text { practices }\end{array}$ & 5.53 & 1.36 & 0.73 \\
\hline WDB & $\begin{array}{l}\text { My office often obtains useful knowledge } \\
\text { from well-defined behaviors }\end{array}$ & 5.93 & 1.17 & 0.81 \\
\hline WDC & $\begin{array}{l}\text { Our office complements the suite of well- } \\
\text { defined components technologies with } \\
\text { other technologies }\end{array}$ & 5.59 & 1.36 & 0.81 \\
\hline
\end{tabular}

All measures of the survey instrument are developed from the literature. Where appropriate, the manner in which the items were expressed is adjusted to the context of supply chains. The items measure the subjects' responses on a seven-point Likert scale ranging from strongly disagree (1) to strongly agree (7).

To check for potential bias of a single informant, the consistency between the data collected from managers was verified. A Chi-square analysis of the industry distribution of the respondents shows no difference from the industry distribution of all the firms used in the survey. This suggests that there is no non-response bias in the returned questionnaires, consistent with past research [20], [22].

\section{2) Data collection}

Questionnaires were distributed to 15 enterprise information systems, after a revision and consulting with 5 reference scholar and 25 information systems engineer; other comments were aggregated again after revision. A pretest and pilot test were performed to validate the instrument before conducting the survey. A pretest on the questionnaire comprising 7 items was carried out with the help of academic researchers to improve the content and appearance of the instrument, as well as to conduct factors analysis. The survey yielded 112 valid responses. Data in Table II explains the demographics of the sample. Responses from early and late respondents show no significant differences in any variable, indicating that late respondents do not differ from early respondents. This finding also implies an absence of non-response bias.

Table II. Demographic characteristics of the sample

\begin{tabular}{|l|l|l|}
\hline Information systems engineer & $\begin{array}{c}\text { Number of } \\
\text { people }\end{array}$ & Percentage \\
\hline Gender & & \\
\hline Male & 87 & 77.6 \\
Female & 25 & 22.4 \\
\hline Level of education & & \\
\hline High school or less & 10 & 9.0 \\
University (undergraduate) & 71 & 63.4 \\
Graduate or high level & 31 & 27.6 \\
\hline Age & & \\
\hline $21-30$ & 13 & 11.6 \\
$31-40$ & 43 & 38.4 \\
$41-50$ & 37 & 33.0 \\
$51-60$ & 5 & 12.0 \\
61 and older & 5.0 \\
\hline
\end{tabular}

\section{Empirical Results}

A confirmatory technique enables the assessment of constructs' reliability and validity. This study employs AMOS software to perform confirmatory factor analysis (CFA) and path analysis of all constructs (Structure-Behavior Coalescence Design and Modifiability of IoT System). CFA results show that the theoretical model has a good fit. To assess the reliability of the constructs, composite reliability (CR) was used. All of the composite reliability values, ranging from a low of 0.866 to a high of 0.890, exceeded the recommended cut-off value of 0.7 . Average variance extracted (AVE) of constructs exceeds the minimum threshold of 0.5 . Thus, results support convergent and discriminant validity of all constructs.

\section{1) Overall model fit}

The relevant overall fit indices (degrees of freedom (df), Root Mean Square Error of Approximation (RMSEA), goodness of fit index (GFI), adjusted goodness of fit index (AGFI), 
comparative fit index (CFI), non-normed-fit index (NNFI), incremental fit index (IFI) ) for each research model implement by AMOS software appear in Table III.

Table III. Overall models fit

\begin{tabular}{|c|c|c|c|c|c|c|c|c|}
\hline \multicolumn{2}{|c|}{ Overall model fit. } & & & & & & & \\
\hline Chi-square & df & p-Value & RMSEA & GFI & AGFI & CFI & NNFI & IFI \\
\hline 17.766 & 13 & 0.167 & 0.057 & 0.958 & 0.909 & 0.99 & 0.984 & 0.99 \\
\hline
\end{tabular}

The Table III data yields chi-squared values of 17.766 for the samples (13 degrees of freedom (df), $p=.167)$. The chi-squared value alone is insufficient for rigorous assessment of the model's fit. A number of additional indexes, however, can shed light on model fit. The GFI should be close to 0.90, NFI more than 0.90, NNFI more than 0.90, CFI more than 0.9, and RMSEA less than 0.08 [19], [21]. An assessment of the measurement model suggested an acceptable model fit $(\mathrm{GFI}=0.958 ; \mathrm{AGFI}=0.909$; $\mathrm{NNFI}=0.984 ; \mathrm{CFI}=0.990 ; \mathrm{IFI}=0.990 ; \mathrm{RMSEA}=0.057$ ) These fit indexes yield values that support a good model fit for the data set.

The results of testing the hypothesized structural equation model screenshot of the AMOS software are shown in Fig. 8.

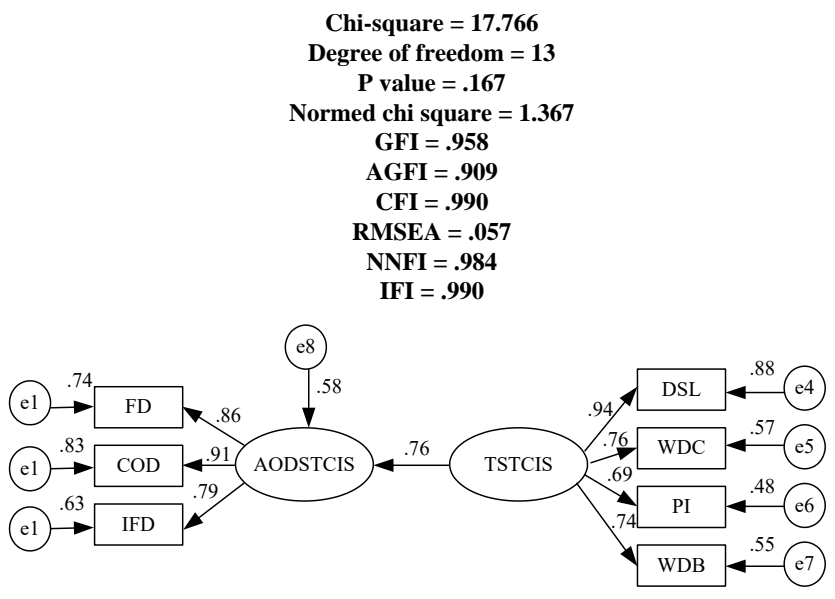

Fig. 8 results of testing the hypothesized structural equation model

\section{2) Measurement model fit}

The measurement model outputs appear in Table 4-1. All 7 standardized loadings are high and have t-values that are significant ( $\mathrm{p} \mathrm{b}$ 0.01). The average variance extracted (AVE) shows the degree of shared representation of the indicators with the constructs. The lowest values for variance extracted are 0.622 for the sample. To investigate the constructs' discriminant validity, Fornell and Larcker [18] suggest observing whether the average variance extracted is greater than the square of the construct's correlations with other factors. Reliability estimates for each construct using Cronbach's alpha [17] and composite reliabilities all exceed the requisite threshold (0.70). All standard errors are small and acceptable. Thus, all indicators relate to their specified constructs, thereby confirming the postulated relationships. All shared variances extracted are acceptable as they exceed the recommended 0.50 value [16], [18]. Overall, the measurement model statistics provide support for the survey instrument's acceptable psychometric properties. Therefore, results support the validity of the structural model.

\section{3) Structural model fit}

The results involve the analyses of the causal paths hypothesis in the structural model. The models support all three hypotheses. Framework Diagram positively effects on Modifiability Home Care IoT System (HCIS); Component Operation Diagram positively effects on Modifiability Home Care IoT System (HCIS); Interaction Flow Diagram positively effects on Modifiability Home Care IoT System (HCIS)

\section{CONCLUSIONS}

Modifiability improvement is a key factor in the successful Home Care IoT System (HCIS) systems development. Disciplined system layering, published interfaces, well-defined components, and well-defined behaviors are four main factors to enhance the testability of HCIS.

Structure-Behavior Coalescence (SBC) design emphasizes the integration of systems structure and systems behavior of a system. SBC design contains three fundamental diagrams: a) framework diagram, b) component operation diagram, and c) interaction flow diagram. Structure-Behavior Coalescence approach uses three fundamental diagrams: a) framework diagram, b) component operation diagram, and c) interaction flow diagram to accomplish the design of HCIS.

Through framework diagram, Structure-Behavior Coalescence design of HCIS demonstrates tremendous effects of disciplined system layering. Through component operation diagram, Structure-Behavior Coalescence design of HCIS demonstrates large effects of well-defined components and published interfaces. Through interaction flow diagram, Structure-Behavior Coalescence design of HCIS demonstrates tremendous effects of well-defined behaviors.

Structural equation modeling (SEM) refers to a diverse set of unrelated computer algorithms and statistical methods that fit networks of constructs to data. Lastly, we use SEM to verify that Structure-Behavior Coalescence design is really be able to enhance the Modifiability of HCIS.

\section{References}

[1] Buhalis, D., "Marketing the Competitive Destination of the Future," Tourism Management, 2000, pp.97-116.

[2] Chao, W. S., General Systems Theory 2.0: General Architectural Theory Using the SBC Architecture, CreateSpace Independent Publishing Platform, 2014.

[3] Chao, W. S., Variants of SBC Process Algebra: The Structure-Behavior Coalescence Approach, CreateSpace Independent Publishing Platform, 2015.

[4] Chao, W. S., System: Contemporary Concept, Definition, and Language, CreateSpace Independent Publishing Platform, 2016. 
[5] Chao, W. S., Generalized SBC Process Algebra for Communication and Concurrency: The Structure-Behavior Coalescence Approach, CreateSpace Independent Publishing Platform, 2016.

[6] Evans, M.W. et al., Software Quality Assurance \& Management, Wiley-Interscience, 1987.

[7] Gharajedaghi, J., Systems Thinking: Managing Chaos and Complexity: A Platform for Designing Business Architecture, Morgan Kaufmann, 2011.

[8] Hoare, C. A. R., Communicating Sequential Processes, Prentice-Hall, 1985.

[9] Kendall, K. et al., Systems Analysis and Design, 8th Edition, Prentice Hall, 2010.

[10]Milner, R., Communication and Concurrency, Prentice-Hall, 1989.

[11]Milner, R., Communicating and Mobile Systems: the $\pi$-Calculus, 1st Edition, Cambridge University Press, 1999.

[12] Paskaleva, K., "Enabling the Smart City: The Progress of E-City Governance in Europe," International Journal of Innovation and Regional Development, 2009, pp.405-422.

[13] Pressman, R. S., Software Engineering: A Practitioner's Approach, 7th Edition, McGraw-Hill, 2009.

[14] Scholl, C., Functional Decomposition with Applications to FPGA Synthesis, Springer, 2010.

[15] Sommerville, I., Software Engineering, 8th Edition, Addison-Wesley, 2006.

[16]Bagozzi, R.P., \& Yi, Y., On the evaluation of structural equation models. Journal of the Academy of Marketing Science, 16, 1988, 74-94.

[17] Cronbach, L.J., Coefficient alpha and the internal structure of tests. Psychometrik,16,1951., 297-333.

[18] Fornell C., and Larcker D.F., Evaluating structural equation models with unobservable variables and measurement error, Journal of Marketing Research, 18 (1), 1981, 39-50.

[19] Hair J.F., Anderson R.E., Tatham R.L., and Black W.C., Multivariate data analysis with readings, 1998, (4th ed), Prentice Hall; New Jersey.

[20] Kishen Iyengar., Jeffrey R. Sweeney., Ramiro Montealegre Kishen., Information technology use as a learning mechanism: the impact of it use on knowledge transfer effectiveness, absorptive capacity, and franchisee performance, MIS Quarterly Vol. 39 No. 3, pp. 615-641/September 2015

[21] Joreskog K.G., and Sorbom D., LISREL 8: Structural equation modeling with the SIMPLIS command language, 1993, Scientific Software International; Chicago.

[22] Weil P., The relationship between investment in information technology and firm performance: A study of the value manufacturing sector, Information Systems Research 3, 1992, 307-333.

[23] Oseikhuemen Davis Ojie, Reza Saatchi, Principal Component Analysis of the Modified Clinical Test of Sensory Interaction in Healthy Adult Humans, pp.
125-142, WSEAS Transactions on Biology and Biomedicine, Volume 17, 2020

[24] Aygun Guliyeva Eldar, Problems of Financial and Investment Support Modelling of the Regional Social and Economic Development, WSEAS Transactions on Business and Economics, pp. 741-752 Volume 17, 2020

[25] Kariman Ramzy El Helow, Abdel-Badeeh M. Salem, Are Artificial Intelligence (AI) and Machine Learning (ML) Having an Effective Role in Helping Humanity Address the New Coronavirus Pandemic? pp. 119-124, WSEAS Transactions on Biology and Biomedicine, Volume 17, 2020

[26] Tahera Parvin, Ariful Islam, Pankaj Kumar Mondal, Md. Haider Ali Biswas, Discrete Type SIR Epidemic Model with Nonlinear Incidence Rate in Presence of Immunity, pp. 104-118, WSEAS Transactions on Biology and Biomedicine, Volume 17, 2020

\section{Contribution of Individual Authors to the Creation of a Scientific Article (Ghostwriting Policy) \\ William S. Chao conceived and designed the} Architecture-Oriented HCIS Model.

Shuh-Ping Sun and William S. Chao performed the Modifiability experiments of HCIS and wrote the paper."

\section{Creative Commons Attribution License 4.0} (Attribution 4.0 International, CC BY 4.0)

This article is published under the terms of the Creative Commons Attribution License 4.0

https://creativecommons.org/licenses/by/4.0/deed.en_US 